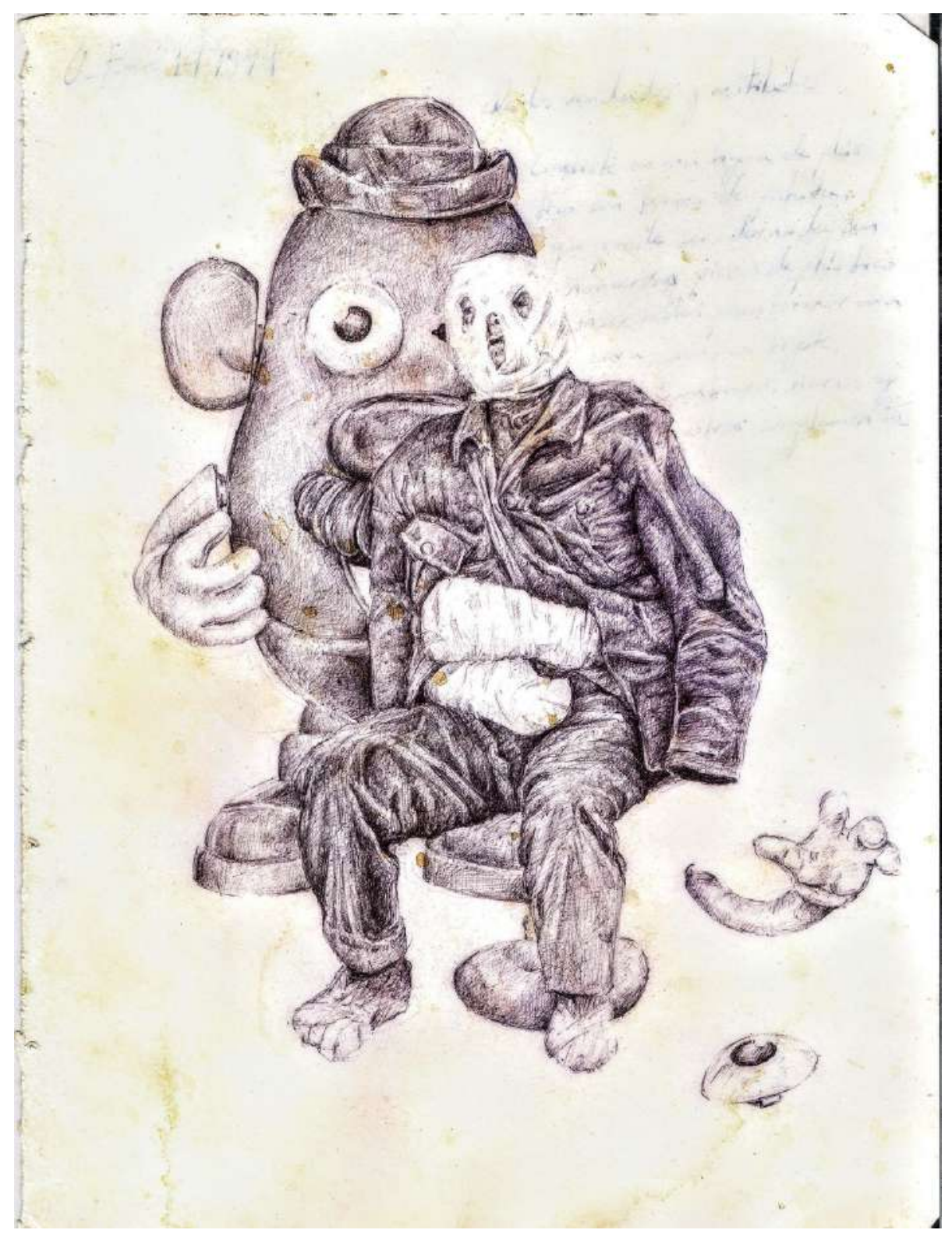

Sebastián Zea Quintero

Sin título

De la serie Del juego y el hombre Lápiz sobre papel 2017

Medellín 


\title{
Desinstitucionalización del sistema de partidos en Bogotá, Colombia (1994-2019). Representación y responsiveness ${ }^{*}$
}

\author{
Yessika Lorena Vásquez González (Colombia)**
}

\section{Resumen}

Este artículo examina, de manera exploratoria, el efecto de la desinstitucionalización del sistemadepartidosen la democracialocal, particularmente, en la representación y la responsiveness en Bogotá. Las investigaciones sobre sistema de partidos en Colombia concluyen que en los últimos años se ha evidenciado una progresiva desinstitucionalización, sin embargo, tal literatura ha prestado poca atención al efecto de la desinstitucionalización en los gobiernos locales. Por ello, se busca responder a las siguientes preguntas: ¿cuáles son las estrategias utilizadas por los partidos para conectar con la ciudadanía?, ¿de qué manera tiene lugar la representatividad y la responsiveness? A partir de una amplia revisión teórica, se analizan datos electorales y estudios empíricos, examinando las estrategias que desarrollan los partidos en Bogotá, en las elecciones a Alcaldía y Concejo. Se concluye que existen dinámicas electorales diferentes: en las elecciones a la [206] Alcaldía se evidencia un fuerte personalismo, donde las organizaciones partidistas tienen un papel secundario; en la dinámica electoral al Concejo se particulariza la representación y la atención de demandas a través de redes delimitadas. En ambos casos hay una menguada capacidad de responsiveness.

\section{Palabras clave}

Instituciones Políticas; Sistema de Partidos; Representación Política; Política Local; Responsiveness.

Fecha de recepción: noviembre de 2019

- Fecha de aprobación: abril de 2020

\footnotetext{
* Este artículo se deriva de un proceso de investigación doctoral entre 2009 y 2013, y en la investigación Dispositivos de participación y movilización ciudadana: su incidencia en la calidad de la democracia a nivel urbano. El caso de Bogotá, financiada por la Universidad de Bogotá Jorge Tadeo Lozano. Agradezco los comentarios y oportunas sugerencias realizadas por el Comité Editorial de la revista Estudios Políticos, así como a los pares evaluadores anónimos.

** Politóloga. Magíster en administración y gerencia pública. Doctorada en América Latina Contemporánea y en Teoría Política, Teoría Democrática y Administración Pública. Profesora titular del Programa de Ciencia Política y Gobierno, Departamento de Ciencia Política y Relaciones Internacionales, Universidad de Bogotá Jorge Tadeo Lozano. Correo electrónico: lorena.vasquezg@ utadeo.edu.co - Orcid: 0000-0003-2147-4363 - Google Scholar: https://scholar.google.com/ citations?hl=es\&user=eTS-fcsAAAAJ
} 


\title{
Cómo citar este artículo
}

Vásquez González, Yessika Lorena. (2020). Desinstitucionalización del sistema de partidos en Bogotá, Colombia (1994-2019). Representación y responsiveness. Estudios Políticos (Universidad de Antioquia), 58. pp. 206-231. DOI: 10.17533/udea.espo.n58a10

\section{Party System Deinstitutionalization in Bogota, Colombia (1994-2019). Representation, and Responsiveness}

\begin{abstract}
This article explores the effect of the deinstitutionalization of the party system on local democracy, particularly on representation and responsiveness in Bogotá. Research on the Colombian party system concludes that a progressive deinstitutionalization has been evident in recent years; however, such literature has paid little attention to the effect of deinstitutionalization at the local level. For this reason, this article seeks to answer the following questions: What are the strategies used by political parties to connect with citizens? How do representativeness and responsiveness take place? This illustrates the strategies used by political parties in Bogota during the elections for mayor and local government. From an extensive theoretical review, electoral data and empirical studies are analyzed, examining the strategies developed by the parties in Bogotá, in the elections for Mayor and Council. This article concludes that there are different electoral dynamics. During the mayoral elections, there is evidence of strong personalism and political parties have a secondary role. In the elections for local Council, the representation and attention to demands take place through delimited electoral and clientelist networks. In both cases, there is a diminished capacity for responsiveness.
\end{abstract}

\section{Keywords}

Political Institutions; Party System; Political Representation; Local Politics; Responsiveness. 


\section{Introducción}

El fundamento de la democracia es la relación entre ciudadanos y gobernantes. Los primeros son los encargados de generar demandas y preferencias expresando su apoyo a través del voto, o mediante diversas estrategias que buscan explícitamente influir en los procesos de gobierno; los segundos - partidos y líderes políticos-, agregan las preferencias en el marco de procedimientos institucionales y toman las decisiones (Urbinati, 2006). En el modelo de democracia representativa, la relación entre representantes -autoridades de gobierno y partidos políticos- y ciudadanos tiene lugar, básicamente, en el marco de procesos electorales, siendo los partidos agentes fundamentales de canalización de intereses (Manin, 1998).

En el esquema democrático representativo es esencial la relación entre intereses — demandas ciudadanas - y resultados — decisiones políticas-, para ello, un elemento clave es que los ciudadanos manifiesten sus necesidades hacia los gobiernos y que estos, a su vez, sean receptivos a tales demandas -responsiveness - . Un gobierno es receptivo — responsive - a las demandas de los ciudadanos si lleva adelante políticas en línea con las preferencias señaladas por sus ciudadanos. Para estos autores, las encuestas de opinión, [208] diversas formas de acción política directa —manifestaciones, protestas, entre otras-, incluso las elecciones, son mecanismos de manifestación de tales preferencias, las cuales se convierten posteriormente en «señales» hacia los gobiernos (Manin, Przeworski y Stokes, 1999, pp. 8-10).

En un sistema de partidos desinstitucionalizado, cuyas organizaciones partidistas cuentan con un débil afianzamiento en la sociedad, así como vínculos programáticos e ideológicos difusos, cabe preguntarse cómo tiene lugar tanto la representación como la responsiveness. Si bien se han efectuado estudios que analizan la desinstitucionalización del sistema de partidos y sus efectos en la democracia en gobiernos nacionales (Mainwaring, 2018b; Mainwaring y Bizzarro, 2018), son pocas las investigaciones orientadas a examinar la desinstitucionalización de partidos, la democracia y la responsiveness en el escenario local. Este artículo busca contribuir a este cuerpo de literatura, analizando el caso de Bogotá, una ciudad que ha experimentado una transformación significativa de su sistema de partidos, y aunque ha surgido un debate social muy intenso respecto a este tema, no ha habido un correlato por parte de la literatura científica para abordarlo con suficiente sistematicidad. Así, el artículo busca responder a las siguientes 
preguntas: ¿cuáles son las estrategias de los partidos para conectar con los electores?, ¿qué tipo de representatividad tiene lugar en el marco de contextos de sistemas de partidos desinstitucionalizados?, ¿cuáles son las características de la responsiveness? Para ello se analizarán las dinámicas electorales, tanto para el alcalde mayor como para el Concejo, en el caso de Bogotá, utilizando revisión documental.

\section{Responsiveness y desinstitucionalización del sistema de partidos. Discusión teórica y conceptualización}

Una de las características clave de la democracia es la capacidad de respuesta continúa del gobierno — responsiveness - a las preferencias de sus ciudadanos, las cuales son consideradas como políticamente iguales (Dahl, 1971, p. 1). De ahí que, junto con la accountability, la responsiveness sea una variable central para examinar la calidad de la democracia. A diferencia de la accountability, la responsiveness no es una dimensión procedimental, sino que engloba al proceso democrático en su conjunto, a la relación agente-principal y alude a la satisfacción de los ciudadanos con el resultado político, cuestión por la cual los estudios empíricos en esta materia son más escasos y revisten de mayor complejidad, sin que haya consenso respecto a los indicadores para dar cuenta de esta variable (Morlino 2007).

Responsiveness hace alusión a la receptividad de partidos y líderes políticos respecto a las demandas de la ciudadanía, receptividad que se traduce en políticas, programas y decisiones de gobierno (Kang y Powell, 2010; Dahl, 1998). El tipo de responsiveness practicado por los diferentes políticos en el ejercicio de gobierno tiene que ver con la posición del partido al que pertenece sobre cómo materializar la relación entre señales y políticas, en tal caso la variable ideológica es central (Linde y Peters, 2018). Los representantes deben responder, ser receptivos — responsive-, a los intereses de los electores, sin embargo, este es precisamente el reto de la relación agente-principal, que los representantes actúen orientados bajo el mejor interés público (Pitkin, 1967). Más que una cuestión normativa, el desafío en los estudios empíricos está en identificar cómo la responsiveness tiene lugar, lo que sin duda contribuye a desentrañar la calidad de la representación, de la participación y, por último, de la democracia.

Los procesos de democratización son posibles en la medida en que existan sistemas de partidos que representen los intereses de los ciudadanos (Mainwaring y Scully, 1995). En contextos de sistemas de partidos 
desinstitucionalizados es probable que haya mayor inestabilidad, tanto de los miembros del sistema de partidos como en la competencia interpartidaria, también respecto a las preferencias ideológicas y programáticas de los partidos (Mainwaring, 2018a). La incertidumbre electoral facilita el surgimiento de políticos outisders, muchos de ellos con poca experiencia; en consecuencia, se incrementa la inestabilidad política y la poca continuidad de las políticas públicas debido a que los horizontes de tiempo son más limitados y no hay organizaciones partidistas que permitan una unidad programática. Igualmente, puede ser proclive a tener conflictos con el parlamento, debido a la dificultad de formar mayorías estables. La posibilidad de efectuar accountability -a través del proceso electoral- y responsiveness es reducida. Todo lo anterior puede conducir a allanar prácticas de clientelismo y corrupción (Mainwaring, 2018b, p. 101).

Scott Mainwaring, al reexaminar los estudios sobre institucionalización del sistema de partidos (Mainwaring y Scully, 1995; Mainwaring y Torcal, 2005), realiza ulteriores aportes a fin de identificar nuevas variables sobre la medicion de este fenómeno. Señala que la institucionalización supone que los actores políticos se comportan de manera estable, con expectativas claras y estables sobre otros actores, lo que a su vez permite crear un entorno organizacional e institucional, cuyas interacciones son establecidas y conocidas por todos. La institucionalización genera un escenario de predictibilidad de comportamientos, tanto de élites políticas como de votantes y demás actores, que da lugar a una mayor previsibilidad al futuro. Las características de institucionalización son: i) estabilidad de los miembros del sistema de partidos; ii) baja volatilidad electoral; y iii) vínculos ideológicos y programáticos de los partidos con sus votantes son ciertamente estables. En el primer caso, los partidos principales son estables y continúan siendo claves elección tras elección; en caso de que surjan nuevos partidos de manera frecuente se estaría dando lugar a la desinstitucionalización. Por su parte, la volatilidad da información clave sobre la regularidad de la competición partidista. Por último, el anclaje del vínculo programático e ideológico alude al grado de enraizamiento del partido en la sociedad y las conexiones existentes entre votantes y partidos (Mainwaring, Bizzarro y Petrova, 2018).

Además de lo anterior, es útil revisar la propuesta de definir el grado de institucionalización del sistema de partidos en función de tres criterios generales: i) nivel de volatilidad; ii) vínculo programático o ideológico entre los votantes y los partidos; y iii) grado de personalismo (Mainwaring y Torcal, 
2005). Como puede apreciarse, los dos primeros fueron retomados, mientras que el último se considera un factor que incide en la institucionalización, pero no como variable central (Mainwaring, Bizzarro y Petrova, 2018). El personalismo tiene que ver con cómo la motivación del voto puede estar precedida por las características personales de los candidatos, más que por las opciones ideológicas que personifican y su vínculo con un partido. Resulta útil, a efectos del presente artículo, integrar el personalismo, ya que la responsiveness tiene que ver con la recepción de demandas de la ciudadanía, pero también con la manera en que se conectan políticos y votantes, y cómo esta conexión permite la canalización de demandas.

En el caso de la variable personalismo, es clave destacar que esta agudiza la desinstitucionalización, dado que profundiza la inestabilidad de la interacción en el sistema partidista. En el caso de un personalismo extremo los partidos tienen poco control sobre quién accede a los cargos públicos y las afiliaciones a los partidos son intrascendentes; así, la competencia electoral obedecería al papel de los individuos más que de los partidos. Los partidos serían irrelevantes para la mayoría de los votantes (Mainwaring, Bizzarro y Petrova, 2018, p. 18).

Vinculando los planteamientos anteriormente enunciados con el análisis de un sistema de partidos desinstitucionalizado en el escenario local, como el caso de Bogotá, es posible adelantar algunas hipótesis: en primer lugar, en contextos de sistemas de partidos desinstitucionalizados, como el caso de Bogotá, hay una baja estabilidad de las organizaciones partidistas, llevando al surgimiento de movimientos o partidos nuevos, los cuales ganan un porcentaje sustancial de votos; en segundo lugar, surgen por ello políticos outsiders, en ocasiones críticos, al menos de manera discursiva, con las prácticas políticas tradicionales; en tercer lugar, dada la limitada identificación partidista, así como la poca congruencia ideológica al interior de los partidos, hay una alta volatilidad electoral, al estar frente a organizaciones partidistas débiles se depende esencialmente de la figura de los líderes políticos personalismo-; en cuarto lugar, la responsiveness — la receptividad a las demandas ciudadanas - tine lugar en el proceso electoral bajo programas amplios y muchas veces difusos en términos ideológicos; por último, los políticos outsiders que llegan a la Alcaldía Mayor, al no contar con mayorías en el Concejo, encuentran un entorno conflictivo para llevar adelante su programa de gobierno. 


\section{Explorando la representación en Colombia: del hermetismo a la fragmentación}

\section{Desinstitucionalización del sistema de partidos en Colombia}

Al analizar los partidos tradicionales y el sistema de partidos en Colombia resultan evidentes las consecuencias del Frente Nacional: i) debilitamiento de la identidad de partido y del vínculo ideológico con los votantes; ii) incremento de la competencia intrapartido y posterior aumento de facciones al interior de estos; iii) mutación e intensificación del clientelismo; y iv) bloqueo a la entrada a terceras fuerzas (Leal, 2005; Gutiérrez, 2007). Todas ellas están estrechamente relacionadas y dieron como resultado el inicio de una progresiva desinstitucionalización del sistema de partidos en Colombia.

El menoscabo de la identidad partidaria está estrechamente relacionado con el efecto del fraccionalismo intrapartidista. Las fracciones dentro de los partidos tradicionales se convirtieron en redes de influencia con un alto grado de autonomía, lo que minó la coordinación y unidad al interior de los partidos. Autonomía que se exacerbó con la Constitución de 1991. El clientelismo fue

[212] el resultado de los dos fenómenos: la pérdida de la identidad partidaria y la «milimetría política» que apoyaba su engranaje en la lealtad electoral regional y municipal, ello se vio favorecido por el incremento de los recursos del Estado. El clientelismo en Colombia ha sido la manera frecuente, en particular, desde el Frente Nacional, con que los líderes políticos tradicionales han conectado con las bases sociales. El menoscabo del vínculo ideológico entre votantes y partidos, la desinstitucionalización de los partidos y la manera de acceder a la distribución de bienes públicos — cargos públicos, contratos, servicios públicos, entre muchos otros- se produce a cambio de votos efectivos; así, el «saqueo del Estado» se convirtió en un lugar común, tanto para políticos como para votantes (Leal y Dávila, 1994; Pizarro, 2002; Gutiérrez, 2007). Para los políticos es una forma «segura» de llegar al poder y para los ciudadanos es, tal vez, la única manera de acceder a servicios del Estado.

La Constitución de 1991 buscó facilitar y promover un proceso de apertura política. El objetivo, más que aumentar la representatividad de los partidos políticos tradicionales, apuntaba al ingreso de nuevos partidos y movimientos que dieran voz a los sectores sociales excluidos hasta ese 
entonces. Primó la denominada «lógica de la incorporación» (Pizarro y Bejarano, 2007), estableciendo una normativa laxa de cara a integrar nuevas fuerzas políticas y sociales. Se pasó de un fraccionalismo intrapartidista a un faccionalismo personalista, donde los partidos tradicionales han ido perdiendo poder, dando lugar a un sistema todavía influido por estos, toda vez que de allí han salido diferentes fuerzas políticas personalistas, pero cuyas organizaciones cada vez son más débiles. Lo anterior subsiste de manera paralela con unas terceras fuerzas con alta fragmentación — de izquierda, partidos cristianos, regionales, indígenas, afros, entre otros-. Tras la atomización generada por la Constitución de 1991, la reforma de 2003 buscó incentivar la reagrupación de partidos para disminuir su número y fortalecer la gobernabilidad, estableciendo requisitos más exigentes para la creación de partidos, entre otros (Hoyos, 2007). La reforma de 2003, si bien limitó la atomización partidista, no ha logrado consolidar los partidos y, en consecuencia, el sistema de partidos.

Colombia pasó de ser catalogada como una democracia con instituciones partidistas en proceso de fortalecimiento, a tener un sistema de partidos desinstitucionalizado (Albarracín, Gamboa y Mainwaring, 2018). Teniendo en cuenta las tres variables arriba señaladas (Mainwaring, Bizzarro y Petrova, 2018), en las últimas décadas se ha producido un cambio profundo ha llevado al declive de los partidos políticos tradicionales, que sin desaparecer han dado lugar al frecuente surgimiento de múltiples movimientos y organizaciones partidistas. Como consecuencia, la volatilidad electoral es elevada. Al no haber partidos políticos fuertes, frecuentemente es difícil formar mayorías o minorías sólidas y estables que hagan posible que los gobiernos cuenten con respaldo político en el legislativo o que exista una oposición capaz de ejercer su papel. Así, la posibilidad de lograr mayorías legislativas a cambio de beneficios en las decisiones políticas — contratos, cuotas burocráticas, entre otros - es una opción que se ha vuelto recurrente tanto en el ámbito nacional como en los departamental y municipal (Albarracín, Gamboa y Mainwaring, 2018, p. 246).

Por su parte, los vínculos ideológicos y programáticos entre los partidos y sus votantes son débiles. La descentralización política promovió el acceso a recursos y competencias administrativas de entes locales, lo que favoreció autonomía frente a las organizaciones partidistas. La debilidad partidista conllevó la atomización y competencia de estas organizaciones, las cuales, 
teniendo acceso a recursos, fortalecieron sus posiciones clientelares. El clientelismo, en la actualidad, cuenta con redes más descentralizadas y atomizadas. Ante el surgimiento de políticos que luchan por estas redes, la competencia entre ellos ha aumentado y el vínculo entre políticos y redes clientelares es cada vez más débil, son intercambios de corto plazo. La relación entre políticos locales y nacionales es menos jerárquica e institucionalizada (Albarracín, Gamboa y Mainwaring, 2018, p. 246).

\subsection{Desinstitucionalización del sistema de partidos en Bogotá}

El declive de los partidos tradicionales en Colombia se agudiza en Bogotá. Estudios sobre partidos y elecciones en Bogotá evidencian esta problemática (Gutiérrez, 1995; García, 2002; 2003; Barrero, 2015; Barrero y Ortega, 2015), señalando la alta volatilidad electoral, el surgimiento de nuevos partidos y movimientos — tanto para las elecciones de alcalde como para las de Concejo-, la baja identificación partidista y la aparición de candidatos outsiders - especialmente para la Alcaldía - que buscan desmarcarse de los partidos, especialmente de los tradicionales, creando así sus propios movimientos.

Desde 1994 hasta 2015, en las elecciones a la Alcaldía de Bogotá se ha apreciado un alto nivel de personalismo, en muchos casos, con candidatos inscritos por firmas y mostrando, al menos de manera discursiva, su distancia respecto a los partidos tradicionales. Ello ha conllevado a una volatilidad electoral que disminuyó en las elecciones de 2003. Como puede apreciarse en la gráfica 1, la volatilidad electoral en las elecciones de alcalde entre 2000 y 2011 es especialmente alta, tuvo una disminución significativa para las elecciones de 2003, donde el partido Polo Democrático Independiente - luego Polo Democrático Alternativo-, dio cierta estabilidad a la competencia partidista, para luego nuevamente incrementarse en las de 2007.

Tal fenómeno de candidatos y alcaldes outsiders contrasta con un Concejo cuya volatilidad ha venido disminuyendo desde el año 2000 (Barrero, 2015), con representación significativa de partidos tradicionales o de facciones derivadas de ellos, especialmente del Partido Liberal. Como puede apreciarse en la tabla 1, la volatilidad es significativamente menor comparada con las elecciones de alcalde. En el periodo 2007-2011 la volatilidad en las 
elecciones de alcalde casi duplicó a la del Concejo. Esta diferencia sustancial entre la volatilidad de las elecciones puede ser resultado de que en el caso del Concejo $-y$ como se verá más adelante, las Juntas Administradoras Locales (JAL) — la dinámica electoral será diferente. Los candidatos se apoyan en plataformas partidistas, como respuesta a las reglas introducidas por la reforma política de 2003. Son organizaciones partidistas débiles, con un alto individualismo, pero con una volatilidad electoral moderada, en muchos casos, siguiendo patrones clientelares que vinculan a los partidos con sus bases. En el caso de las elecciones a la Alcaldía Mayor, especialmente desde 1994, se ha evidenciado una conexión personalista y carismática entre el candidato - y el alcalde-con los electores, sin que esté mediado por una sólida organización partidista —a excepción de la votación de 2007- El voto de opinión es clave para ganar los comicios, promoviendo una representación política más personalista, con candidatos provenientes de movimientos políticos independientes a los partidos tradicionales. En consecuencia, se mantiene, en cierta medida, una constante: por una parte, ejecutivos pertenecientes a terceras fuerzas y, en algunos casos, con un discurso transformador, y Concejos con un perfil anclado, en ocasiones, a prácticas políticas tradicionales, en términos clientelares (Gutiérrez, 1995; García, 2002; 2003).

Gráfica 1. Volatilidad electoral en la elección de alcaldes Bogotá, 2000-2011.

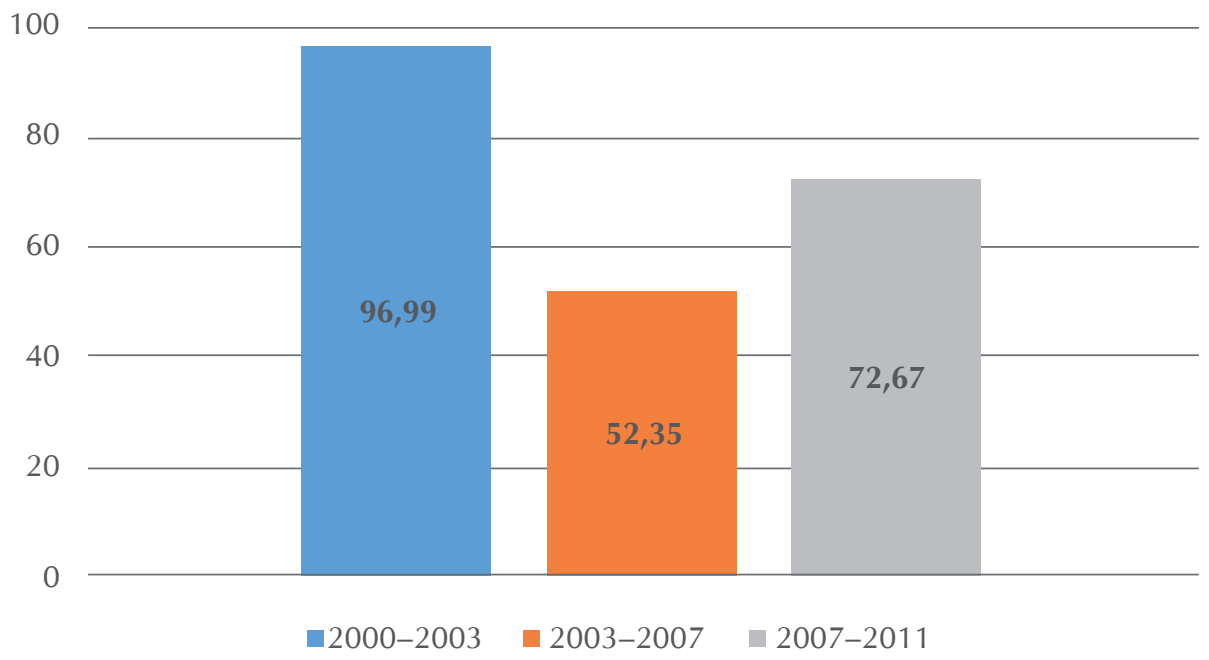

Fuente: elaboración propia a partir de Fredy Barrero (2015). 
Tabla 1. Volatilidad electoral en las elecciones al Concejo de Bogotá, 2000-2011.

\begin{tabular}{|c|c|}
\hline Periodo & Volatilidad \\
\hline $2000-2003$ & 64.48 \\
\hline $2003-2007$ & 50.95 \\
\hline $2007-2011$ & 34.40 \\
\hline $2011-2015$ & 24.57 \\
\hline
\end{tabular}

Fuente: elaboración propia a partir de Fredy Barrero y Felipe Ortega (2015, p. 277).

Respecto al vínculo programático o ideológico entre los votantes y los partidos, no hay muchos estudios sobre esta cuestión en Bogotá. Un análisis muy interesante aborda la identidad partidista y su vínculo con el comportamiento electoral a través de una proxy, la simpatía partidista (Angulo, 2016). En él se explora la relación entre la simpatía partidista y la intención de voto en Bogotá. El estudio evidencia que las preferencias de voto en Bogotá son significativamente inestables, así como que tanto la simpatía partidista como la intención de voto presentan cambios en el corto plazo. Según su evidencia empírica, más de $50 \%$ de los ciudadanos suelen cambiar su simpatía partidista después de las elecciones (p. 92), lo que puede evidenciar una débil identidad partidista. A juicio de la autora, para los comicios de 2011 puede señalarse que los electores de Bogotá, en su mayoría, no tienen simpatía partidista, los ciudadanos no se sienten identificados, en general, con ningún partido o movimiento político, y la intención de voto es más volatil que tal simpatía. En tal caso, se evidencia que el voto personalista es clave en las elecciones de Bogotá, donde las características y el perfil del candidato son más importantes que el rótulo partidista al momento de tomar una decisión de voto. Este personalismo se agudizara por la ausencia de mecanismos que promuevan una articulación efectiva entre los programas de los candidatos y sus partidos, y el frecuente surgimiento de outsiders que crean sus propios movimientos. Este fenómeno se observa de forma más asidua en las elecciones a alcalde. Así, al parecer la reforma de 2003, aunque logró detener la atomización del sistema de partidos, no ha logrado confrontar el personalismo, de hecho, los incentivos al voto personalista, aun al interior de las organizaciones partidistas, prevalecen. 
En relación a la débil articulación entre los programas de candidatos y sus partidos, existen partidos que al momento de dar el aval al candidato no señalan una orientación programática a aquel para la elaboración de su programa, como el Partido de la U. Hay otros partidos, como el Liberal, que aunque sí establecen principios programáticos a sus candidatos, una vez revisado el programa de determinado candidato no se encuentra que su contenido refleje tales principios. A su juicio, «No se encontró indicio alguno de que las directivas ejerzan control alguno, siquiera mínimo, de lo que ofrecen sus candidatos» (Losada, 2012, p. 127).

\section{Pugna por la representación en Bogotá}

\subsection{La tensión entre la Alcaldía Mayor y el Concejo}

Es en las elecciones a la Alcaldía Mayor y al Concejo de Bogotá donde se aprecia con más claridad la pugna entre dos tipos de resposiveness y la progresiva desinstitucionalización del sistema de partidos. Por una parte, la figura del alcalde, antipolítico, outsider, que se autodenomina representante por excelencia de los intereses ciudadanos; por otro, el Concejo, que se suele relacionar con los partidos tradicionales y con prácticas proclives al clientelismo. Esto ha tenido consecuencias significativas tanto en la relación entre votantes y partidos políticos como - y de manera más intrincada- en la relación entre Alcaldía y Concejo.

Lo anterior ha conllevado a que, desde la elección popular de alcaldes, habitualmente las mayorías partidistas del Concejo no coincidan con el partido al que pertenece el alcalde de la ciudad. No hay una consistencia entre las preferencias del voto a alcalde y las preferencias para el Concejo y las JAL (Gutiérrez, 2001). Tal inconsistencia ha generado una sugerente contradicción al interior de la dinámica electoral de Bogotá: una ciudad que pareciera contar con una participación electoral crítica y autónoma, y otra anclada al bagaje y a las formas del hacer del clientelismo. Las anteriores dinámicas subsisten de manera paralela y parecen alimentarse la una de la otra.

El voto de opinión - uno de los indudables patrimonios históricos de la ciudad-como puramente reflexivo y autónomo ...] creó un hiato entre dos ciudades que se negaban mutuamente, una naturalpremoderna y la otra cívica-moderna. Ese hiato comenzó como algo externo a la política y a la participación, pero terminó enquistándose en ellas (Gutiérrez, 1998, p. 51). 
Hasta la elección de Antanas Mockus en 1994 el partido mayoritario en el Concejo era, en general, el que también se llevaba la mayor votación para alcalde mayor. Como lo ilustra la tabla 2, en 1990 y 1992 fue evidente la correspondencia entre partido ganador en la Alcaldía y partido mayoritario en el Concejo. Esta dinámica fue truncada con la llegada de Mockus. «A partir de 1994, la Alcaldía Mayor y el Concejo aparecen como espacios enfrentados [...]. Se empieza a consolidar una suerte de tecnocracia en el ámbito del ejecutivo, que se contrapone a un Concejo que se proyecta como el espacio de las prácticas políticas tradicionales» (García, 2002, p. 202). Incluso, desde 1994 el proceso de gobierno ha estado signado, en la mayor parte, por constantes tensiones entre el ejecutivo y el Concejo, el cual, debido a la dificultad de formar coaliciones mayoritarias en el Concejo por parte del alcalde, ha generado que sus negociaciones estén mediadas por las promesas de burocracia y contratos por parte del ejecutivo como instrumento para lograr apoyo a su gestión, o directamente por una relación conflictiva.

En el caso de los dos periodos de Mockus, la relación entre Concejo y alcalde se caracterizó por la indiferencia o el bloqueo mutuo. Con un Concejo con mayoría del Partido Liberal, su relación fue «traumática» (Bromberg y Contreras, 2005, p. 29). En 1995 el alcalde presentó su programa de Plan de Desarrollo al Concejo, los concejales quisieron adicionar modificaciones sin la suficiente discusión ni aprobación por parte del alcalde, razón por la cual Mockus decidió aprobarlo por decreto. El carácter antipolítico del alcalde y el hecho de no pertenecer a partidos tradicionales generó una creciente tensión que el alcalde manejó utilizando el capital político obtenido por el apoyo ciudadano:

El hecho de haber llegado a la más importante Alcaldía del país sin deberles favores electorales a ninguno de los partidos [...] le da un importante capital político. Por eso Mockus se puede dar lujos que les estuvieron prohibidos a sus antecesores, amarrados por unas maquinarias clientelistas y en buena medida responsables del estado de postración en que se encuentra la ciudad (El Tiempo, 1995, junio 4).

En el periodo de Enrique Peñalosa (1998-2000) hubo un vínculo más orientado a la transacción, lo que llevó a que su relación fuera más fluida (Jiménez, 2007; Bromberg y Contreras, 2005). Nuevamente, el Partido Liberal —antiguo partido de Peñalosa — tuvo mayorías: «logró parte de esa eficiencia a través de un hábil manejo del Concejo, lo cual supuso mantener 
Desinstitucionalización del sistema de partidos en Bogotá, Colombia (1994-2019)...

Tabla 2. Partidos ganadores a la Alcaldía y al Concejo de Bogotá, 1988-2015.

\begin{tabular}{|c|c|c|c|c|c|}
\hline \multirow[b]{2}{*}{ Año } & \multicolumn{3}{|c|}{ Alcaldía } & \multicolumn{2}{|r|}{ Concejo } \\
\hline & Partido ganador & $\begin{array}{c}\% \\
\text { votos }\end{array}$ & \begin{tabular}{|c|} 
Total de \\
candidatos
\end{tabular} & $\begin{array}{c}\text { Partido } \\
\text { mayoritario }\end{array}$ & $\begin{array}{l}\text { \% votos } \\
\text { \# curules }\end{array}$ \\
\hline 1988 & Partido Conservador & $34,9 \%$ & 21 & Partido Liberal & \begin{tabular}{|l}
$45 \%$ \\
9 curules \\
20 concejales elegidos
\end{tabular} \\
\hline 1990 & Partido Liberal & $65,6 \%$ & 11 & Partido Liberal & $\begin{array}{l}45 \% \\
11 \text { curules } \\
20 \text { concejales elegidos }\end{array}$ \\
\hline 1992 & Partido Liberal & $54,1 \%$ & 17 & Partido Liberal & \begin{tabular}{|l|}
$49 \%$ \\
15 curules \\
28 concejales elegidos \\
\end{tabular} \\
\hline 1994 & $\begin{array}{l}\text { Otros partido o } \\
\text { movimientos }\end{array}$ & $64,5 \%$ & 3 & Partido Liberal & \begin{tabular}{|l|}
$51,5 \%$ \\
18 curules \\
34 concejales elegidos
\end{tabular} \\
\hline 1997 & $\begin{array}{l}\text { Otros partidos o } \\
\text { movimientos (Liberal) }\end{array}$ & $48,6 \%$ & 15 & Partido Liberal & \begin{tabular}{|l|}
$42,8 \%$ \\
19 curules \\
40 concejales elegidos \\
\end{tabular} \\
\hline 2000 & $\begin{array}{l}\text { Alianza Social } \\
\text { Independiente (ASI) - } \\
\text { Asociación de Usuarios } \\
\text { de Créditos del UPAC } \\
\text { (Anupac) - Visionarios }\end{array}$ & $44,7 \%$ & 11 & Partido Liberal & $\begin{array}{l}31,3 \% \\
11 \text { curules } \\
42 \text { concejales elegidos }\end{array}$ \\
\hline \multirow{2}{*}{2003} & \multirow{2}{*}{$\begin{array}{l}\text { Polo Democrático } \\
\text { Independiente (PDI) }\end{array}$} & \multirow{2}{*}{$49,3 \%$} & \multirow{2}{*}{14} & PDI & \begin{tabular}{|l|}
$13,7 \%$ \\
8 curules \\
45 concejales elegidos \\
\end{tabular} \\
\hline & & & & Partido Liberal & \begin{tabular}{|l}
$12,8 \%$ \\
8 curules
\end{tabular} \\
\hline \multirow[t]{2}{*}{2007} & \multirow{2}{*}{$\begin{array}{l}\text { Polo Democrático } \\
\text { Alternativo (PDA, antes } \\
\text { PDI) }\end{array}$} & \multirow[t]{2}{*}{$46,8 \%$} & \multirow[t]{2}{*}{6} & PDA & \begin{tabular}{|l}
$22,5 \%$ \\
11 curules \\
45 concejales elegidos \\
\end{tabular} \\
\hline & & & & $\begin{array}{l}\text { Cambio } \\
\text { Radical }\end{array}$ & $\begin{array}{l}21,5 \% \\
11 \text { curules }\end{array}$ \\
\hline \multirow[t]{2}{*}{2011} & \multirow[t]{2}{*}{ Movimiento Progresistas } & \multirow[t]{2}{*}{$33,2 \%$} & \multirow[t]{2}{*}{9} & Partido de la $U$ & \begin{tabular}{|l}
$17,8 \%$ \\
8 curules \\
45 concejales elegidos \\
\end{tabular} \\
\hline & & & & Progresistas & $\begin{array}{l}17,7 \% \\
8 \text { curules }\end{array}$ \\
\hline \multirow[t]{2}{*}{2015} & \multirow[t]{2}{*}{ Recuperemos Bogotá } & \multirow[t]{2}{*}{$33,2 \%$} & \multirow[t]{2}{*}{9} & $\begin{array}{l}\text { Cambio } \\
\text { Radical }\end{array}$ & $\begin{array}{l}15 \% \\
9 \text { curules } \\
45 \text { concejales elegidos } \\
\end{array}$ \\
\hline & & & & $\begin{array}{l}\text { Centro } \\
\text { Democrático }\end{array}$ & $\begin{array}{l}11 \% \\
6 \text { curules }\end{array}$ \\
\hline
\end{tabular}

Fuente: elaboración propia a partir de Registraduría Nacional del Estado Civil (s. f.). 
vivo el esquema de apoyo político a cambio de contratos y burocracia» (García, 2002, p. 214).

En el siguiente periodo de gobierno —Mockus (2001-2003) — se revivieron las tensas relaciones entre el Concejo y la Alcaldía Mayor que caracterizaron el primer mandato de Mockus. Esto generó un gran desgaste entre las relaciones del ejecutivo y el legislativo de la ciudad, que supuso un menoscabo para la gobernabilidad de la ciudad. Este mutuo bloqueo institucional y la imposibilidad de llegar a acuerdos, sin duda, tuvieron efectos negativos en el proceso decisional y en los proyectos de mayor envergadura para la ciudad.

De 2003 a 2011 uno de los partidos mayoritarios en el Concejo fue el mismo que el del alcalde, el Polo Democrático Independiente (PDI). Este punto es significativo ya que desde 1997 ningún partido había logrado mayoría absoluta en el Concejo. El gobierno de Luis Eduardo Garzón (2004-2007) se destacó por su concertación con las diversas fuerzas políticas, incluyéndolas en la conformación del gabinete, lo que facilitó la gobernabilidad. De la concertación entre el alcalde y el Concejo, reflejada en la conformación del gabinete, cabía esperar el aminoramiento de la pugna entre estos dos [220] entes, como efectivamente sucedió (Bromberg y Contreras, 2005, p. 32). Sin embargo, Garzón encontró más críticas en su propio partido, el PDI. Un concejal miembro del PDI afirmó en una entrevista que «[Garzón] ha conciliado con las viejas prácticas de la política del chantaje, las presiones y las negociaciones [...]. De alguna manera muy pragmática y muy cómoda concilió con esas prácticas» (El Tiempo, 2005, febrero 12).

Posteriormente, en el gobierno de Samuel Moreno (2008-2011) se pasó de la concertación a la connivencia. El alcalde contó con una coalición mayoritaria que le permitió la aprobación del Plan de Desarrollo y los cupos de endeudamiento, entre otros proyectos clave. ${ }^{1}$ De las negociaciones clientelistas que ya se veían venir al inicio de su periodo de gobierno (Bromberg, 2008, agosto 6) se pasó a uno de los mayores escándalos de corrupción en la ciudad —el Carrusel de la Contratación-, en el cual estuvieron investigados 17 concejales y el alcalde mayor: "La investigación acerca del "carrusel"

\footnotetext{
${ }^{1}$ Esta coalición fue posible gracias al acuerdo con las bancadas de los partidos de la U, Liberal, Conservador, Convergencia Ciudadana, Alas-Equipo Colombia y Opción Verde. La negociación estuvo mediada por el reparto de comisiones en diferentes contrataciones de la ciudad (Acuña y Rodríguez, 2016, marzo 21).
} 
ha dejado condenas y ha sembrado dudas sobre el gobierno distrital en su conjunto y sobre toda la clase política relacionada con la capital» (Acuña y Rodríguez, 2016, marzo 21).

El siguiente alcalde, Gustavo Petro (2012-2015), fue quien denunció cuando era senador el Carrusel de la Contratación. Petro no logró consolidar coaliciones mayoritarias en el Concejo, tanto el partido de la $U$ como Cambio Radical y el Partido Liberal, hicieron oposición, a la que posteriormente se unieron algunos miembros del Partido Verde y el Partido Conservador. Las relaciones fueron tensas desde un inicio y el conflicto fue escalando a lo largo del periodo de gobierno (Semana, 2014, abril 4), y temas claves como la modificación excepcional del Plan de Ordenamiento Territorial y la modernización tributaria no fueron aprobados (Bogotá Cómo Vamos, 2015).

Finalmente, en el segundo periodo de Peñalosa en la Alcaldía (20162019) hubo una parte significativa del Concejo que apoyó su gestión, logrando una coalición mayoritaria con 34 concejales, excepcional si se revisan los periodos de gobierno anteriores (Bogotá Cómo Vamos, 2015). Se lograron aprobar la mayoría de proyectos clave, entre otros, los cupos de endeudamiento.

Si bien durante algunos periodos de gobierno — correspondientes a Luis E. Garzón (2004- 2007) y a Samuel Moreno (2008-2011) — el partido del alcalde tuvo mayorías en el Concejo, esto no ha sido la generalidad desde 1994. Como puede apreciarse en esta revisión de la relación alcalde-Concejo en Bogotá, se ha pasado de la tensión —Mockus I y ॥- a la negociación y concertación —Peñalosa I y ॥, Garzón-, por la confrontación abierta -Petro- y, por supuesto, por la connivencia, dando lugar a procesos de corrupción —Moreno-. Se puede señalar que en los periodos de negociación y concertación —Peñalosa I y II, Garzón, incluso Moreno- se lograron coaliciones, resta por estudiar cuáles fueron las características de tales coaliciones y por qué se lograron mantener.

En un sistema de partidos desinstitucionalizado es más difícil obtener el apoyo del legislativo o conseguir mayorías estables duraderas, en este caso, Concejo (Mainwaring, 2018b). Este apoyo puede ser débil, surgiendo así conflictos entre ejecutivo y legislativo. La falta de mayorías, a priori, puede conducir a que la negociación para lograr coaliciones mayoritarias en el Concejo, a fin de tener mayor gobernabilidad por parte del alcalde, sea más 
ardua, con dificultad para lograr acuerdos estables. Así pues, la capacidad de ejercer veto por parte del Concejo, sus funciones en el control político al ejecutivo y de aprobación de temas claves como el presupuestal, cupo de endeudamiento, Plan de Desarrollo o Plan de Ordenamiento Territorial, llevan a una necesaria transacción entre estos dos entes. Como se demuestra, alcaldes críticos hacia prácticas políticas tradicionales han encontrado mayor conflicto en el Concejo, con evidentes dificultades para llevar a cabo su programa de gobierno y con consecuencias claras en la gobernabilidad: «Si el ejecutivo tiene urgencia, y su capacidad de espera es casi nula, los congresistas pueden pedir compensaciones particularistas a cambio de aprobar las leyes» (Gutiérrez, 2003, p. 24). Esto puede ser extrapolado al gobierno local en el caso de Bogotá. Es preciso examinar cuál es la naturaleza de esos intercambios.

\subsection{Ese gran desconocido. Concejo y responsiveness}

Al preguntar a los ciudadanos qué instituciones, según su percepción, realizan acciones para mejorar su calidad de vida, 28\% señaló que la Alcaldía y solo 5\% que el Concejo (Bogotá Cómo Vamos, 2017). Buena parte del electorado en la ciudad se ha concentrado en la figura del alcalde, atribuyendo al ejecutivo la responsabilidad de los resultados del ejercicio de gobierno

[222] (Pizano, 2003); por el contrario, la percepción de la gestión de los concejales de la ciudad parece casi inexistente:

Muchos de los que habitan la Capital desconocen que existe una escala Distrital donde la cabeza es el alcalde pero que es seguida por una Corporación que se dedica al control y vigilancia de la administración de turno debatiendo acuerdos que fungen como la hoja de ruta para el desarrollo, bienestar y avance de la ciudad.

[...] De todo lo anterior se desprenden varias consecuencias: [...] (2) La participación ciudadana se ve limitada al voto y no trasciende al control social que debe ejercer el ciudadano sobre la gestión de los concejales. (3) No hay una verdadera rendición de cuentas por parte de los legisladores (Contrial, 2015, mayo 8).

Este desconocimiento de las funciones del Concejo y su importancia para el gobierno de la ciudad se refleja en que para las elecciones de 2011 en Bogotá más de $90 \%$ de los encuestados no recordaban por cuál candidato al Concejo habían votado, 95\% no sabía si ese candidato había salido elegido y más de 90\% consideraban que el Concejo no los representaba (Hurtado, García y Copete, 2013). Por lo tanto, parece que la relación entre el Concejo y el 
ciudadano de Bogotá es distante, lo que, en principio, limitaría los procesos de representación política, en términos de accountability y responsiveness. En caso de que los ciudadanos busquen promover determinados temas en la agenda, pueden no ver a los concejales de la ciudad como autoridades que puedan ser receptivas a sus demandas o que puedan canalizarlas de manera efectiva — responsiveness - . De la misma manera, en caso de crisis por determinados temas en la ciudad, no se aprecia la responsabilidad de los concejales. Así pues, surge la pregunta: ¿cómo se representan los intereses de los ciudadanos si hay una débil conexión entre los partidos políticos y los electores?

\subsection{De la representación al clientelismo. Vínculos entre partidos y votantes}

El denominado voto de opinión y su consiguiente distanciamiento con las prácticas políticas tradicionales, parece ser generalizado en la ciudad (Pizano, 2003); no obstante, aunque este voto de opinión independiente ha empezado a surgir con fuerza en Bogotá, no es generalizable a toda la ciudad, ni implica que las dinámicas políticas de la ciudad, en efecto, hayan tenido una transformación profunda, desligándose de prácticas clientelares (Barreto, 2010).

Lo que a priori puede parecer una mayor institucionalización de los partidos, en el caso de Concejo, evidencia partidos con mayor capacidad organizativa en lo local y lo barrial, que en muchos casos opera a través del personalismo de los candidatos y de sus redes electorales, articuladas a un candidato determinado, no necesariamente a un partido. Esto ha llevado a que tanto para las elecciones de Concejo como para las JAL predominen dinámicas personalistas y, en algunos casos, clientelares. Más que vínculos ideológicos o programáticos, se advierten vínculos personalistas, difusos y, en ocasiones, estratégicos para la obtención de bienes públicos entre los representantes políticos y la ciudadanía.

Estudiando la dinámica electoral del Concejo y las JAL se aprecia un funcionamiento más robusto de las organizaciones partidistas de cara a ganar elecciones. Tal robustez, sin embargo, no se equipara con la institucionalización. Este comportamiento más «sólido» de los partidos se puede explicar por dos cuestiones: la primera, debido a la reforma política de 2003 y la Ley de Bancadas que estableció nuevas reglas en el sistema de 
partidos, favoreciendo la reagrupación de los partidos. Antes de la reforma de 2003, los partidos repartían avales de manera indiscriminada a quienes lo solicitaran y demostraran que tenían cierto caudal electoral (Pizarro, 2002). Este panorama no ha cambiado del todo. Conscientes de las altas probabilidades que tienen muchos políticos de base de acceder a cargos de elección popular contando únicamente con sus redes electorales, estos buscan el aval dentro del «mercado» de partidos: «[...] no es el partido el que escoge el candidato, sino éste quien escoge cuál etiqueta partidaria le conviene» (Losada, 2012, p. 128). Debido al creciente descrédito de los partidos tradicionales frente a los electores, muchos de los candidatos buscan marcar distancia hacia la maquinaria política tradicional, lo que lleva a que buena parte de ellos en las campañas electorales se consideren, por ejemplo, liberales $y$, a la vez, independientes. ${ }^{2}$

La desinstitucionalización y la ruptura de las adscripciones partidistas de los candidatos hacia sus electores, en términos de la identidad, se evidencia cuando los ciudadanos que votan por una lista perdedora a la JAL, avalada por determinado partido, no se consideran representados por una lista de ese mismo partido que sí obtiene una curul (Bromberg y Contreras, 2005). Es decir, los votantes se sienten identificados por determinado candidato no por [224] el partido.

La reforma de 2003 obligó a los partidos a realizar un proceso de selección de candidatos más juicioso, contrastando con la estrategia de repartir avales de manera indiscriminada anotada anteriormente. Con las nuevas reglas, algunos partidos han institucionalizado más el proceso que otros, en ciertos casos los partidos han sido más abiertos y democráticos, ${ }^{3}$ realizando consultas internas para la selección de candidatos, aunque se reconoce que la utilización de este mecanismo es ínfima (Rodríguez, 2015).

¿Cuál es, entonces, el vínculo entre el ciudadano y el partido o el candidato? La segunda explicación al funcionamiento más robusto de los partidos en el Concejo y la JAL, comparado con la elección de alcalde mayor, tiene que ver con su relación con las bases sociales y sus estrategias

\footnotetext{
${ }^{2}$ Considerando la pérdida de legitimidad de los partidos, los candidatos deben mostrar un discurso antipartido ante los electores, lo que les otorga un aura de mayor confianza, pero de cara al proceso político, siguen estado dentro de las redes de los partidos tradicionales (Gutiérrez, 2002, pp. 47-55).

${ }^{3}$ Véase el caso particular de la consulta del Partido Verde en 2015 para la selección de sus candidatos a ediles para conformar listas para JAL en Bogotá (Rodríguez, 2015).
} 
electorales encaminadas a maximizar su participación en los comicios. Las denominadas microempresas electorales tenían — todavía - su fundamento en que los candidatos partían del supuesto de que buena parte - si no el total- de los votos obtenidos dependían más de su propio esfuerzo, de sus tácticas electorales y de su financiamiento de campaña que de la capacidad organizacional del partido (Albarracín, Gamboa y Mainwaring, 2018).

Se denomina microempresas electorales a grupúsculos, bien al interior de los partidos o como movimientos o partidos nuevos, que buscan representación en los legislativos o ejecutivos a partir de redes electorales restringidas e identificables encabezadas por un líder político. Tal líder político puede ser, por ejemplo, candidato al Concejo y a su vez apoyase en líderes barriales e incipientes líderes políticos territoriales, como candidatos a la JAL o líderes de Juntas de Acción Comunal, para articularse a las bases sociales y obtener los votos necesarios para obtener escaños (Pizarro, 2002).

Así se forma una red que vincula líderes y votantes. Muchas de estas redes funcionan a partir de lógicas clientelares, indispensables para obtener votantes «cautivos» y para garantizar el triunfo en las elecciones. Al correlacionar los votos obtenidos en determinados barrios y la inversión aprobada para estas zonas desde la JAL — georreferenciación electoral—, existe una alta incidencia entre el éxito electoral de los ediles y el direccionamiento de inversión hacia aquellos barrios donde obtuvieron mayor votación (Bromberg y Contreras, 2005, pp. 187-195). La lógica que subyace parece ser direccionar el gasto o los recursos de la localidad hacia aquellas zonas o sectores en donde estos representantes obtuvieron más votos.

Los anteriores hallazgos parecen evidenciar la particularización de la representación en la elección a Concejo o JAL, donde la red electoral de ediles o concejales está relativamente delimitada. De esta manera, aseguran su elección con un número no muy alto de votos y los ciudadanos garantizan cierto acceso a los servicios y bienes públicos. La lógica clientelar determina quién esta «dentro» de la red y quién está «fuera». Los que están dentro, es decir, los ciudadanos cuyos candidatos han logrado un cargo a Concejo o JAL, pueden acceder a bienes y servicios del Estado que son distribuidos de manera discrecional — desde cupos en colegios, servicio de salud, hasta cargos públicos, contratos, entre otros- Los que están fuera, aquellos que decidieron no votar o cuyo candidato perdió, tendrán mayores dificultades para acceder a los mismos bienes y servicios (Gutiérrez, 1998, p. 119). 
Los representantes políticos — concejales o ediles— que están a cargo de distribuir recursos públicos tienen incentivos para utilizar la personalización y delimitación de redes electorales o el clientelismo como mecanismo para llegar al Concejo o a la JAL, de esta manera se aseguran de obtener los votos que necesitan para lograr las curules. Por su parte, los ciudadanos que integran redes electorales o clientelares tienen incentivos para seguir perteneciendo a ellas, es la forma en que logran acceder a servicios que tal vez de otro modo no podrían. Esta puede ser la forma en la que opera la responsiveness en el ámbito local, particularizando demandas ciudadanas.

\section{Conclusiones}

El análisis permitió apreciar una evidente desinstitucionalización del sistema de partidos de Bogotá, exponiendo la debilidad de las organizaciones partidistas, el surgimiento de nuevos partidos y movimientos —en particular, para la elección a alcalde-, el endeble vínculo programático e ideológico entre votantes y partidos, el cual se expresa en una alta volatilidad electoral, y un elevado personalismo. Tal desinstitucionalización tiene consecuencias en la gobernabilidad, pero también en la representación y la responsiveness.

[226] Relativo a la gobernabilidad, la literatura señala que bajo contextos de desinstitucionalización del sistema de partidos es probable que se presenten conflictos frecuentes entre ejecutivo y legislativo. A este respecto, se ha evidenciado consecuencias significativas en términos de las relaciones alcalde-Concejo en Bogotá. Las organizaciones partidistas están conformadas por candidatos que cuentan con agendas e intereses particulares. Así, ante la fragmentación de la representación y el bajo arraigo ideológico, surgen acuerdos inestables, generando una clara dificultad para establecer coaliciones mayoritarias en el Concejo. La posibilidad de veto del Concejo lleva a la necesidad de realizar acuerdos entre el ejecutivo y la corporación; sin embargo, la diferencia en la estrategia electoral —una, en ocasiones antipolítica (alcalde), la otra, en muchos casos, utilizando las prácticas tradicionales (Concejo) - puede conducir a frecuentes conflictos entre estos dos entes, como en efecto se apreció en el periodo 1994-2019, o llevar a acuerdos tácitos y estratégicos donde el intercambio de favores políticos parece beneficiar la gobernabilidad, pero ir en contra de los discursos contra la política tradicional del alcalde.

Frente a la desinstitucionalización del sistema de partidos, las elecciones de alcalde mayor y de Concejo han adoptado estrategias diferenciadas 
que reflejan distintas maneras de hacer efectiva la representatividad y la responsiveness. En el caso de los candidatos a alcalde, ante la inexistencia de organizaciones partidistas ancladas en la sociedad a través de vínculos ideológicos, promueven una conexión personalista con los electores, basado en un discurso crítico hacia los partidos políticos tradicionales y sus prácticas políticas. Materializan un discurso transformador que lleva a movilizar, en muchos casos, a votantes independientes. Por otra parte, la dinámica electoral del Concejo y las JAL promueve redes electorales delimitadas, caracterizadas por la generación de incentivos para pertenecer a dichas redes, en muchos casos clientelares, a fin de acceder a bienes y servicios del Estado. Así se particulariza la representación y la atención de demandas.

En este escenario surgen preguntas tales como: ihay incentivos para atender demandas más universales, menos focalizadas?, ¿qué pasa con los ciudadanos que no pertenecen a tales redes? , ¿cómo se atienden sus demandas? Aunque la reforma de 2003 intentó fortalecer la institucionalización del sistema, en general, los mayores logros tuvieron que ver con la reagrupación de partidos, manteniendo intacto el personalismo y el clientelismo, así como la innegable debilidad ideológica de las organizaciones partidistas.

Las anteriores dinámicas electorales se traducen en débiles vínculos de representación entre partidos y ciudadanos, con una menguada capacidad de responsiveness. Representación significa actuar de acuerdo con los mejores intereses del público (Pitkin, 1967). Las elecciones suponen representatividad, pero este ejercicio solo es posible en la medida en que existan partidos que articulen preferencias y las representen, y que estos actúen en favor del interés de los electores. Los programas electorales darían información sobre las políticas y los ciudadanos elegirían los que consideren más conveniente. De igual manera, los ciudadanos pueden manifestar sus necesidades hacia los gobiernos y que estos sean receptivos a tales demandas — responsiveness-. Ante la ausencia de partidos institucionalizados se debilita el ejercicio de la representación, teniendo claras consecuencias en la responsiveness.

Es pertinente que en la agenda de investigación sobre estas cuestiones se explore de manera más sistemática cuáles son las consecuencias de la desinstitucionalización del sistema de partidos en la calidad de la democracia, en la legitimidad de los gobiernos y en el sistema político, y de manera particular abordar de qué manera la relación entre señales — demandas ciudadanasy resultados — decisiones políticas - tiene lugar en los ámbitos nacional y 
subnacional en Colombia. Resulta acuciante emprender investigaciones de este tipo en un momento histórico en donde se ha incrementado la movilización social y las estructuras de representación parecen insuficientes para canalizar tales demandas.

\section{Referencias bibliográficas}

1. Acuña, Fabián Alejandro y Rodríguez, Juan Guillermo. (2016, marzo 21). Samuel Moreno, el carrusel y la corrupción en Bogotá. Razón Pública. Recuperado de https://razonpublica.com/samuel-moreno-el-carrusel-y-la-corrupcion-en-bogota/

2. Albarracín, Juan; Gamboa, Laura \& Mainwaring, Scott. (2018). Deinstitutionalization without Collapse: Colombia's Party System. In: Mainwaring, Scott (ed.). Party Systems in Latin America: Institutionalization, Decay, and Collapse (pp. 227-254). Cambridge: Cambridge University. https://doi. org/10.1017/9781316798553.009

3. Angulo, María Camila. (2016). Intención de voto y simpatía partidista en Bogotá. Colombia Internacional, 86, pp. 81-106. https://doi.org/10.7440/ colombiaint86.2016.03

4. Barrero, Fredy. (2015). Participación electoral en Bogotá 1998-2014. Registraduría Nacional del Estado Civil. Recuperado de https://www.registraduria. gov.co/IMG/pdf/Libro___Analisis_de_la_participaciion_c.pdf

5. Barrero, Fredy y Ortega, Felipe. (2015). Bogotá y sus mutaciones electorales. En: Barrero, Fredy (ed.). Elecciones regionales 2015: los retos de un país en camino hacia la paz (pp. 269-292). Bogotá, D. C.: Konrad Adenauer Stiftung.

6. Barreto, Antonio. (2010). El voto de opinión en Bogotá: una mirada crítica. Revista Análisis Político, 69, pp. 66-78.

7. Bogotá Cómo Vamos. (2015) Concejo cómo vamos. Informe de monitoreo, seguimiento y evaluación del Concejo de Bogotá. Il semestre 2015. Bogotá, D. C.: Bogotá Cómo Vamos.

8. Bogotá Cómo Vamos. (2017). Encuesta de Percepción Ciudadana 2017. Bogotá, D. C.: Bogotá Cómo Vamos.

9. Bromberg, Paul. (2008, agosto 6). Bogotá: un gobierno sin marca. Razón Pública https://www.razonpublica.com/index.php/regiones-temas-31/74-bogotungobierno-sin-marca.html

10. Bromberg, Paul y Contreras, Yency. (2005). Olivos y Aceitunos: ¿Todos son Uno? El Concejo de Bogotá y el Plan de Desarrollo Distrital 2004-2008. En: Bogotá Sin Indiferencia. Una mirada ciudadana sobre el Plan De Desarrollo Distrital 20042008 (pp. 27-32). Bogotá, D. C.: Guadalupe.

11. Corporación para el Control Social (Contrial). (2015, mayo 8). Comunicado N. ${ }^{\circ}$ 8: 45 concejales también deciden en Bogotá. Recuperado de http://contrial.co/ comunicado-n-8-45-concejales-tambien-deciden-en-bogota/ 
12. Dahl, Robert A. (1971). Polyarchy. Participation and Opposition. New Haven: Yale University.

13. Dahl, Robert A. (1998). On Democracy. New Haven: Yale University.

14. Dávila, Andrés y Delgado, Natalia. (2002). La metamorfosis del sistema político colombiano: ¿clientelismo de mercado o nueva forma de intermediación? En: Gutiérrez, Francisco (ed.). Degradación o cambio. Evolución del sistema político colombiano (pp. 319-356). Bogotá, D. C.: Norma.

15. El Tiempo. (1995, junio 4). Mockus contra todos. Recuperado de https:// www.eltiempo.com/archivo/documento/MAM-338604

16. El Tiempo. (2005, febrero 12). Se concilió con el chantaje: De Roux. Recuperado de https://www.eltiempo.com/archivo/documento/MAM-1634457

17. García Sánchez, Miguel. (2002). La política bogotana, un espacio en recomposición (1982-2001). En: Gutiérrez, Francisco (ed.). Degradación o cambio. Evolución del sistema político colombiano (pp. 35-84). Bogota, D. C.: Norma.

18. García Sánchez, Miguel. (2003). ¿Ciudadanía avergonzada? Democracia local y construcción de ciudadanía en Bogotá. Bogotá, D. C.: Universidad de los Andes, CESO.

19. Gutiérrez, Francisco. (1995). Tendencias de cambio en el sistema de partidos: El caso de Bogotá. Análisis Político, 29, pp. 73-82.

20. Gutiérrez, Francisco. (1998). La ciudad representada. Política y conflicto en Bogotá. Bogotá, D. C.: TM, lepri.

21. Gutiérrez, Francisco. (2001). ¿Se ha abierto el sistema político colombiano? Una evaluación de los procesos de cambio (1970-1998). América Latina Hoy, 27. pp. 189-215. https://doi.org/10.14201/alh.2739

22. Gutiérrez, Francisco (ed.). (2002). Historias de democratización anómala. El Partido Liberal en el sistema político desde el Frente Nacional hasta hoy. En: Degradación o cambio. Evolución del sistema político colombiano (pp. 25-78). Bogotá, D. C.: Norma.

23. Gutiérrez, Francisco. (2003). Los tiempos de las involuciones democráticas. Programa de Estados en Crisis. Documentos de Trabajo, 25. Recuperado de http:// eprints.Ise.ac.uk/28266/2/WP25FG.pdf

24. Gutiérrez, Francisco. (2007). ¿Lo que el viento se llevó? Los partidos políticos y la democracia en Colombia 1958-2002. Bogotá, D. C.: Norma.

25. Hoyos, Diana. (2007). Entre la persistencia y el cambio: reconfiguración del escenario partidista y electoral de Colombia. Bogotá, D. C.: Universidad del Rosario.

26. Hurtado, David; García, Diana y Copete, Andrés. (2013). Las vicisitudes del capital social de Colombia. Bogotá, D. C.: Fundación Restrepo Barco.

27. Jiménez, William G. (2007). Las relaciones Alcaldía-Concejo Distrital: apuntes sobre una gobernabilidad y gobernanza en Bogotá. Polémica, 8, pp. 48-58. 
28. Kang, Shin-Goo \& Powell, Bingham. (2010). Representation and Policy Responsiveness: The Median Voter, Election Rules, and Redistributive Welfare Spending. Journal of Politics, 72 (4), pp. 1014-1028. https://doi.org/10.1017/ S0022381610000502

29. Leal, Francisco. (2005) Colombia: un bipartidismo en crisis. En: Meyer, Lorenzo y Reina, José Luis (eds.). Los sistemas políticos en América Latina (pp. 237268). México D. F.: Siglo xxı.

30. Leal, Francisco y Dávila, Andrés. (1994). Clientelismo. El sistema político y su expresión regional. Bogotá, D. C.: TM, lepri.

31. Linde, Jonas \& Peters, Yvette. (2018). Responsiveness, Support, and Responsibility: How Democratic Responsiveness Facilitates Responsible Government. Party Politics, 26 (3). https://doi.org/10.1177/1354068818763986

32. Losada, Rodrigo. (2012). El papel de la organización partidista en las campañas electorales: dos ciudades y dos departamentos colombianos, 2011. En: Wills, Laura y Batlle, Margarita (eds.). Política y territorio. Análisis de las elecciones subnacionales en Colombia, 2011 (pp. 105-130). Bogotá: PNUD-IDEA-NIMD.

33. Mainwaring, Scott (ed.). (2018a). Party System Institutionalization in Contemporary Latin America. In: Party Systems in Latin America: Institutionalization, Decay, and Collapse (pp. 34-70). Cambridge: Cambridge University. https://doi. org/10.1017/9781316798553.003

34. Mainwaring, Scott (ed.). (2018b). Party System Institutionalization,

[230] Predictability, and Democracy. In: Party Systems in Latin America: Institutionalization, Decay, and Collapse (pp. 71-101). Cambridge: Cambridge University. https://doi. org/10.1017/9781316798553.004

35. Mainwaring, Scott \& Bizzarro, Fernando. (2018). Democratization without Party System Institutionalization: Cross-National Correlates. In: Mainwaring, Scott (ed.). Party Systems in Latin America: Institutionalization, Decay, and Collapse (pp. 102-132). Cambridge: Cambridge University. https://doi.org/10.1017/9781316798553.005

36. Mainwaring, Scott \& Scully, Timothy R. (eds.). (1995). Introduction. Party Systems in Latin America. In: Building Democratic Institutions. Party Systems in Latin America (pp. 1-34). Stanford: Stanford University.

37. Mainwaring, Scott y Torcal, Mariano. (2005). La institucionalización de los sistemas de partidos y la teoría del sistema partidista después de la tercera ola democratizadora. América Latina Hoy, 41, pp. 141-173. https://doi.org/10.14201/ alh. 2442

38. Mainwaring, Scott; Bizzarro, Fernando \& Petrova, Ana. (2018). Party System Institutionalization, Decay, and Collapse. In: Mainwaring, Scott (ed.). Party Systems in Latin America: Institutionalization, Decay, and Collapse (pp. 17-33). Cambridge: Cambridge University. https://doi.org/10.1017/9781316798553 
39. Manin, Bernard. (1998). Los principios del gobierno representativo. Madrid: Alianza.

40. Manin, Bernard; Przeworski, Adam \& Stokes, Susan. (1999). Introduction. In: Przeworski, Adam; Stokes, Susan \& Manin, Bernard (eds.). Democracy, Accountability and Representation (pp. 1-26). Cambridge: Cambridge University. https://doi. org/10.1017/CBO9781139175104.001

41. Morlino, Leonardo. (2007). Explicar la calidad democrática: ¿Qué tan relevantes son las tradiciones autoritarias? Revista de Ciencia Política (Santiago), 27 (2), pp. 3-22. https://doi.org/10.4067/S0718-090X2007000300001

42. Pitkin, Hanna F. (1967). The Concept of Representation. Berkeley: University of California.

43. Pizarro, Eduardo. (2002). La atomización partidista en Colombia: el fenómeno de las microempresas electorales. En: Gutiérrez, Francisco (ed.). Degradación o cambio. Evolución del sistema político colombiano (pp. 357-401). Bogotá, D. C.: Norma.

44. Pizarro, Eduardo \& Bejarano, Ana María. (2007). Political Reform After 1991: What Still Needs to be Reformed? In: Welna, Christopher \& Gallón, Gustavo (eds). Peace, Democracy and Human Rights in Colombia (pp. 167-201). Notre Dame: University of Notre Dame.

45. Pizano, Larissa. (2003). Bogotá y el cambio. Percepciones sobre la ciudad y la ciudadanía. Bogotá, D. C.: lepri, CESO.

46. Registraduría Nacional del Estado Civil. (s. f.). Histórico de resultados. Recuperado de https://www.registraduria.gov.co/-Historico-de-Resultados,3635-.html

47. Rodríguez Pico, Clara Rocío (dir.). La selección de candidatos como mecanismo de democratización de los partidos y movimientos políticos en las elecciones subnacionales del 2015 en Colombia. Registraduría del Estado Civil de Colombia. Recuperado de https://www.registraduria.gov.co/IMG/pdf/La_seleccion_ de_candidatos_como_mecanismo_de_democratizacion.pdf

48. Semaña. (2014, abril 4). La pelea de Petro con el Concejo de Bogotá. Recuperado de https://www.semana.com/nacion/articulo/la-pelea-de-petro-con-elconcejo-de-bogota/405014-3

49. Urbinati, Nadia. (2006). Representative Democracy: Principles and Genealogy. Chicago: Chicago University. https://doi.org/10.7208/ chicago/9780226842806.001.0001 